\title{
When you live with a degenerative illness, no symptoms are mild
}

\author{
Comparing a patient's symptoms to those who are worse off can be far from reassuring, says Beth \\ McHugh
}

\section{Beth McHugh}

I was first given a diagnosis of relapsing remitting multiple sclerosis in my 20s. Four years later, a string of relapses triggered involuntary movements in my arms and legs, and I had pain, visual problems, and debilitating fatigue. When my symptoms were at their peak, I couldn't work as a writer and artist. I couldn't cook for myself, hold my own drinks, or shower without someone in the house. Eventually I had to move back in with my parents. This was devastating, and I felt as if all my worst fears about my illness were coming true.

I have seen several specialists over the years. On one occasion, however, I was told that I didn't qualify for a drug that I was interested in because my symptoms were too mild. These words were devastating to me, although perhaps not for the obvious reason. I didn't mind being told that the drug wasn't suitable-this was probably true. What upset me was my symptoms being categorised in this way.

\section{Mild disease can have a severe impact}

The immediate effect was that I felt belittled. Was I ridiculous to be wasting precious NHS time and resources given my symptoms? I felt stupid for letting these symptoms have such a massive impact on my life. I even worried that my doctor didn't believe that I was ill. Being compared to patients with worse symptoms didn't make me feel better; it made me feel more frightened of the future.

The diagnosis of an incurable degenerative illness had already irrevocably altered the life I imagined for myself and put an ever widening gap between me and "normality." Knowledge of the illness alone cast a shadow over my daily life. Every new symptom took me further away from the future that I hoped for and closer to the one I feared. If the symptoms that had destroyed my life were mild then my future looked bleak.

\section{Consider whether and how to discuss the severity of a disease}

The appointment could have been different if I'd been asked "How do these symptoms affect your life?" as well as "What symptoms are you experiencing?" That way I could have explained how my "mild" symptoms, such as dropping my pen every 30 seconds and changes to my vision, had ended the career I'd spent seven years training for. Textbooks might categorise symptoms on a scale of mild to severe, but that's not always a helpful thing to share with patients. Every new symptom I experience, regardless of severity, has a huge emotional impact on me.

A doctor could suggest that a treatment is inappropriate without referring to a severity scale. For example, I would be happy to hear that the drug I was interested in wasn't right for me because, in the doctor's opinion, the side effects would outweigh the benefits, but that he or she would help me to find one that was more suitable.

When patients have a chronic or incurable illness, they need a good relationship with their doctor. They need to be a team. So please try to avoid comparisons with other patients and, please don't tell patients that they could be worse off. It can be helpful to hear that clinicians will work with patients until they find the right treatment; after all, they are on this journey together for a long time. Fortunately, I was prescribed a different drug, which has been a success and I haven't had a relapse since.



\section{Education into practice}

- Can you think of times when you have discussed disease severity with patients? How did it go?

- Does this article offer you ideas on how better to discuss and distinguish disease and symptom severity, if it is necessary?

- How do you discuss which management options may be more or less suitable with patients, and the way in which you will work with them to find the best one?

- Does this article offer you any other ideas on how to change your practice? 
